\title{
Efectividad del programa "La Era vive feliz sin EDAS" en los conocimientos, actitudes y prácticas sobre el manejo de enfermedades diarreicas agudas en las madres de niños menores de 4 años del Centro Poblado Virgen del Carmen - La Era, 2013
}

\author{
Effectiveness of the program "La Era lives happily without EDAS" knowledge, attitudes and \\ practices of the management of acute diarrhea by mothers of children under age 4 in the Town \\ Center Virgen del Carmen - La Era March to December 2013
}

Marisol Rodríguez Villanueva ${ }^{1}$, Julissa López Pariona², Esther Valencia Orrillo

\section{RESUMEN}

Objetivo: Determinar la efectividad del programa "La Era vive feliz sin EDAS" en los conocimientos, actitudes y prácticas sobre el manejo de enfermedades diarreicas agudas en las madres de niños menores de 4 años del Centro Poblado Virgen del Carmen La Era. Material y Métodos: El estudio fue cuantitativo de diseño preexperimental no probabilístico, porque se trabajó con un solo grupo a quien se le aplicó un pretest y postest, a fin de determinar la efectividad del programa. Resultados: Después de la implementación del programa la mayoría evidencia un nivel conocimiento excelente en el manejo de las EDAS (93.3\%) y solo el 6.7\% tiene un conocimiento regular; además, la mayoría presenta una actitud altamente favorable (86.7\%) frente al manejo de las EDAS. Lo mismo sucede con las prácticas, donde las madres luego del programa en su mayoría practican los ítemes que previenen la aparición de las enfermedades diarreicas agudas. Conclusiones: El programa "La Era vive feliz sin EDAS" fue efectivo en el incremento del nivel de conocimientos, actitudes y prácticas sobre prevención de enfermedades diarreicas agudas en las madres de niños menores de 4 años del Centro Poblado Virgen del Carmen-La Era, 2013.

Palabras clave: Madres o cuidadora, enfermedades diarreicas agudas, rehidratación, deshidratación, sales de rehidratación oral.

\begin{abstract}
Objective: To determine the effectiveness of the program "La Era lives happily without EDAS" in knowledge, attitudes and practices on the management of acute diarrheal diseases (EDAS) of mothers of children under four years of the Town Centre Virgen del Carmen La Era. Material and Methods: The study was quantitative probabilistic no pre experimental design because we worked with one group to which was applied a pre test and post test to determine program effectiveness. Results: After implementation of the program most excellent knowledge level evidence on the management of EDAS (93.3\%) and only $6.7 \%$ have a regular knowledge; moreover, most have a highly favorable (86.7\%) compared to management of EDAS attitude. The same applies to practices where mothers program then practiced mostly items that prevent the occurrence of acute diarrheal diseases. Conclusions: the "La Era lives happily without EDAS" was effective in increasing the level of knowledge, attitudes and practices on prevention of acute diarrheal disease in mothers of children under four years of the Town Centre Virgen del Carmen La Era.
\end{abstract}

Keywords: Mothers or caregiver, acute diarrheal diseases, hydration, dehydration, oral rehydration salts.

\footnotetext{
${ }^{l}$ Licenciada en Enfermería, Jefe Laboratorio, E.A.P, Enfermería. Universidad Peruana Unión, Perú.

${ }^{2}$ Licenciada en Enfermería, Clínica Ricardo Palma, San Isidro, Perú.

${ }^{3}$ Magíster en Salud Pública, Profesora asociada de la E.A.P. Enfermería. Universidad Peruana Unión, Perú.
} 


\section{INTRODUCCIÓN}

Las enfermedades diarreicas constituyen las principales causas de morbilidad y mortalidad en los niños menores de cinco años de los países en desarrollo y, en el Perú, estos dos problemas también son frecuentes y constituyen las dos primeras causas de mortalidad infantil (Benguigui, Bossio, Fernández, 2001). La mortalidad infantil está estrechamente vinculada a la pobreza; debido a ello, los avances en la supervivencia de bebés y niños han sido más lentos en la población de los países pobres y en la población más pobre de los países con más recursos. La mejora de los servicios públicos de salud es un elemento clave, en particular el acceso a agua potable y a un mejor saneamiento. La instrucción, especialmente para las niñas y las madres, puede salvar la vida de muchos niños (Instituto Nacional de Estadística, 2003).

La EDAS afecta principalmente a la población infantil de nuestro país. Su presencia aún es notoria entre las principales causas de morbilidad, especialmente en los ámbitos geográficos de la población en situación de pobreza. Las enfermedades diarreicas agudas son enfermedades infeccionas producidas por virus, bacterias, hongos o parásitos, que afectan principalmente a niños menores de cinco años. Mundialmente causan un promedio 4.6 millones de muertes infantiles anuales, de los cuales el $70 \%$ ocurre por deshidratación, complicación más frecuente y grave de la enfermedad.

Los cuadros diarreicos suelen presentarse frecuentemente en la temporada de verano. De igual forma, el riesgo se corre con el consumo de alimentos contaminados tales como los mariscos y pescados, vegetales y frutas sin lavar, entre otros. La forma más efectiva de prevenir esta enfermedad radica en el correcto lavado de manos que, de forma práctica y económica, evita la transmisión de las enfermedades diarreicas agudas. Asimismo, como otras medidas importantes se debe resaltar el tratamiento y consumo de agua segura, y la cocción de alimentos (Minsa, 2011).

Para concientizar a las madres o cuidadoras de la $5 .^{\circ}, 6 .^{\circ}$ y $7 .^{\text {ma }}$ etapa del centro poblado Virgen del Carmen La Era, del manejo de las enfermedades diarreicas agudas en niños menores de 4 años, se decidió realizar un programa titulado "La Era vive feliz sin EDAS", cuyos objetivos están enfocados en mejorar los conocimientos, actitudes y prácticas en el manejo de las enfermedades diarreicas agudas por las madres o cuidadoras que asisten al centro de salud y que sus niños hayan presentado, al menos, un episodio de enfermedades diarreicas agudas, en el que se aplicó un pretest para hacer un diagnóstico situacional, en base a esos resultados realizamos las sesiones de aprendizaje con sus respectivos talleres de redemostración, al finalizar las 12 sesiones de aprendizaje se aplicó un postest para evaluar si el programa fue efectivo y si las madres mejoraron el nivel de conocimientos, actitudes y prácticas.

\section{MATERIALES Y MÉTODOS}

El presente estudio de investigación es de tipo cuantitativo, de diseño preexperimental no probabilístico, porque se trabajó con un solo grupo de muestra, a quien se le aplicó un pretest y postest, a fin de determinar la efectividad del programa.

La población de estudio estuvo constituida por 40 madres o cuidadoras de niños menores de 4 años que asisten al centro de salud La Era Virgen del Carmen (V, VI, VII etapa) y que sus niños hayan presentado, al menos, un episodio de EDAS en sus diferentes tipos (disentérica, aguda o acuosa). Con fines de estudio, la muestra se seleccionó de forma no probabilística, ya que la muestra fue escogida de acuerdo a criterios de inclusión y exclusión, siendo 30 madres.

En primer lugar, se solicitó un permiso al director del establecimiento La Era para realizar el programa "La Era Vive feliz sin EDAS". Después de haber obtenido el permiso, se seleccionó criterios de inclusión y exclusión a la muestra, teniendo registradas, para iniciar el programa, a 40 madres o cuidadoras durante los meses de noviembre-diciembre a las cuales se les informó sobre el programa a realizarse. El programa empezó el 6 de enero del 2014, la frecuencia de las sesiones fueron una vez por semana, juntamente con las visitas domiciliarias, finalizando el 3 de marzo del mismo año.

Para el análisis, se utilizó el software estadístico para Windows aplicado a las ciencias sociales SPSS 20.0, una vez recolectada la información, se transfirió a la matriz de datos del software estadístico para su respectivo análisis; los resultados obtenidos fueron ordenados en tablas. Se utilizó la prueba de bondad de ajuste de Kolmogorov-Smirnov (K-S), para las actitudes y las prácticas frente al manejo de las EDAS, en la evaluación post, ya que presentan una distribución normal y el coeficiente obtenido (KS) no es significativo $(p>0.05)$. Por tanto, para los análisis estadísticos correspondientes, de estas dos variables, se empleará estadística paramétrica. Sin embargo, el nivel de conocimientos frente al manejo de las EDAS no presenta una distribución normal ( $p$ $<0.05$ ), por lo cual para el análisis de esta variable se utilizará estadísticos no paramétricos. 
Efectividad del programa "La Era vive feliz sin EDAS" en los conocimientos, actitudes y prácticas sobre el manejo de enfermedades diarreicas agudas en las madres de niños menores de 4 años del Centro Poblado Virgen del Carmen - La Era, 2013

\section{RESULTADOS}

Tabla 1

Nivel de conocimiento en el manejo de EDAS, antes y después de la intervención

\begin{tabular}{lllll}
\hline Nivel de conocimiento & $\mathrm{N}^{\circ}$ & Pre intervención & $\mathrm{N}^{\circ}$ & Post intervención \\
\hline Deficiente & 26 & $86.7 \%$ & 0 & $0 \%$ \\
Regular & 3 & $10 \%$ & 3 & $6.7 \%$ \\
Excelente & 1 & $3.3 \%$ & 27 & $93.3 \%$ \\
Total & 30 & $100 \%$ & 30 & $100 \%$ \\
\hline
\end{tabular}

Se observa, en la tabla 1, que la mayoría de las madres o cuidadoras antes de la intervención del programa presenta un nivel de conocimiento deficiente $(86.7 \%)$, y solo el $3.3 \%$ tiene un nivel conocimiento excelente en el manejo de las EDAS.
A diferencia del nivel de conocimiento observado en las madres o cuidadoras después de la intervención, donde la mayoría evidencia un nivel conocimiento excelente en el manejo de las EDAS (93.3\%), y solo el $6.7 \%$ tiene un conocimiento regular.

Tabla 2

Nivel de actitudes en el manejo de EDAS, antes y después de la intervención.

\begin{tabular}{lllll}
\hline Nivel de actitudes & $\mathrm{N}^{\circ}{ }^{\circ}$ & Preintervención & $\mathrm{N}^{\circ}$ & Posintervención \\
\hline Altamente desfavorable & 1 & $3.3 \%$ & 0 & $0 \%$ \\
Desfavorable & 3 & $10 \%$ & 0 & $0 \%$ \\
Favorable & 12 & $40 \%$ & 4 & $13.3 \%$ \\
Altamente favorable & 14 & $46.7 \%$ & 26 & $86.7 \%$ \\
Total & 30 & $100 \%$ & 30 & $100 \%$ \\
\hline
\end{tabular}

La tabla 2 permite apreciar que antes de la implementación del programa, solo el $46.7 \%$ de las madres presentaba una actitud altamente favorable hacia el manejo de las EDAS pero, después de la intervención, la mayoría de las madres o cuidadoras presenta una actitud altamente favorable $(86.7 \%)$.

Tabla 3

Prácticas en el manejo de EDAS, antes y después de la intervención del programa "La Era Vive feliz sin EDAS” en madres o cuidadoras en niños menores de 4 años, en el centro de salud Virgen Carmen la Era.

\begin{tabular}{|c|c|c|c|c|c|c|}
\hline & Ítems & & N. ${ }^{\circ}$ & Pretest & N..$^{\circ}$ & Postest \\
\hline \multirow[t]{2}{*}{1} & \multirow{2}{*}{$\begin{array}{l}\text { ¿Las manos de los miembros de familia se muestran limpias y } \\
\text { uñas cortas? }\end{array}$} & NO & 24 & $80 \%$ & 3 & $10 \%$ \\
\hline & & sí & 6 & $20 \%$ & 27 & $90 \%$ \\
\hline \multirow[b]{2}{*}{2} & \multirow{2}{*}{ ¿Realizas el lavado de manos correctamente? } & NO & 25 & $83.3 \%$ & 9 & $30 \%$ \\
\hline & & sí & 5 & $16.7 \%$ & 21 & $70 \%$ \\
\hline \multirow{2}{*}{3} & \multirow{2}{*}{ ¿Tapas y proteges los alimentos? } & NO & 17 & $56.7 \%$ & 1 & $3.3 \%$ \\
\hline & & Sí & 13 & $43.3 \%$ & 29 & $96.7 \%$ \\
\hline \multirow{2}{*}{4} & \multirow{2}{*}{ ¿Existe la presencia de vectores? } & NO & 5 & $16.7 \%$ & 5 & $16.7 \%$ \\
\hline & & sí & 25 & $83.3 \%$ & 25 & $83.3 \%$ \\
\hline \multirow{2}{*}{5} & \multirow{2}{*}{ ¿Mantienes la cocina limpia y ordenada? } & NO & 11 & $36.7 \%$ & 3 & $10 \%$ \\
\hline & & sí & 19 & $63.3 \%$ & 27 & $90 \%$ \\
\hline \multirow{2}{*}{6} & \multirow{2}{*}{ ¿Las mascotas están fuera de casa? } & NO & 22 & $73.3 \%$ & 15 & $50 \%$ \\
\hline & & sí & 8 & $26.7 \%$ & 15 & $50 \%$ \\
\hline
\end{tabular}


7 ¿Cambias semanalmente el trapito de limpiar la mesa?

8 ¿Almacenas agua en bidones limpios y tapados?

9 ¿Purificas el agua antes de consumirlo?

10 ¿Utilizas el tacho de basura con tapa?

\begin{tabular}{lllll}
\hline NO & 30 & $100 \%$ & 30 & $100 \%$ \\
Sí & 0 & $0 \%$ & 0 & $0.0 \%$ \\
\hline NO & 13 & $43.3 \%$ & 3 & $10 \%$ \\
sí & 17 & $56.7 \%$ & 27 & $90 \%$ \\
\hline NO & 8 & $26.7 \%$ & 0 & $0 \%$ \\
Sí & 22 & $73.3 \%$ & 30 & $100 \%$ \\
\hline N0 & 21 & $70.0 \%$ & 19 & $63.3 \%$ \\
Sí & 9 & $30.0 \%$ & 11 & 36.7 \\
\hline NO & 23 & $76.7 \%$ & 23 & $76.7 \%$ \\
Sí & 7 & $23.3 \%$ & 7 & $23.3 \%$ \\
\hline N0 & 21 & $70 \%$ & 6 & $20 \%$ \\
Sí & 9 & $30 \%$ & 24 & $80 \%$ \\
\hline N0 & 16 & $53.3 \%$ & 10 & $33.3 \%$ \\
sí & 14 & $46.7 \%$ & 20 & $66.7 \%$ \\
\hline
\end{tabular}

Se observa, en la tabla 3 , que la mayoría de las madres antes de iniciar el programa "La Era vive feliz sin EDAS" no practicaba el hábito de tener las manos limpias y las uñas cortadas $(80 \%)$. No obstante, luego de la implementación del programa, se aprecia que solo el $10 \%$ de las madres no practica estas medidas de higiene. De manera similar ocurre con la mayoría de las prácticas que previenen la aparición de las EDAS, donde las madres, luego del programa, practican

\section{DISCUSIÓN}

En nuestra sociedad, y a nivel mundial, las enfermedades diarreicas están ocasionando un aumento acelerado de casos en la población infantil en niños menores de 5 años, produciendo grandes dificultades. En los países subdesarrollados en África, Asia y América Latina estiman una importante causa de morbimortalidad. Por tal motivo, es importante tener en cuenta el diagnóstico de EDAS (Cabrera, 2012).

Al analizar los resultados obtenidos en la investigación, se encontró que antes del programa casi la mayoría de las madres o cuidadoras presenta un nivel de conocimiento deficiente (86.7\%) en el manejo de las EDAS. A diferencia del nivel de conocimiento observado en las madres o cuidadoras después de la intervención, donde la mayoría evidencia un nivel conocimiento excelente en el manejo de las EDAS $(93.3 \%)$ y solo el $6.7 \%$ tiene un de conocimiento regular, en el centro de salud Virgen Carmen la Era (V, VI, VII). Al respecto, los resultados obtenidos el lavado de manos (70\%), el baño corporal diario (96.7\%), lavado de las frutas y verduras $(86.7 \%)$, mantenimiento de la cocina limpia y ordenada (90\%), el mantenimiento del agua en bidones limpios y cubiertos $(90 \%)$, purificación del agua $(100 \%)$, mantenimiento del baño limpio (66.7\%), entre otras prácticas. Sin embargo, no se observan cambios en algunas prácticas como sacar la basura diariamente y el cambio semanal del paño yesley.

por Cabezas (2011) fueron similares a los estudios antes de la intervención educativa, las madres tenían un desconocimiento sobre enfermedades diarreicas agudas en un $80 \%$, luego de la intervención educativa se elevó sus conocimientos en un $95 \%$. Con respecto a las actitudes que presenta las madres o cuidadoras en el manejo de EDAS, antes de la implementación del programa, solo el $46.7 \%$ de las madres presentaba una actitud altamente favorable hacia el manejo de las EDAS pero, después de la intervención, la mayoría de las madres o cuidadoras presenta una actitud altamente favorable $(86.7 \%)$. Por otro lado, Quispe (2012) menciona que el 83.3\% tiene prácticas y actitudes deficientes, estando directamente relacionado con el grado de analfabetismo y primaria incompleta mostrando un porcentaje representativo del $27.8 \%$ y $66.67 \%$ respectivamente. Por lo tanto, el conocimiento influye en las actitudes y la práctica.

En cuanto a las prácticas de las madres o cuidadoras, existen diferencias significativas en el manejo de las EDAS, pues antes de la intervención del programa presentaba una media de 22,83 y después 
Efectividad del programa "La Era vive feliz sin EDAS" en los conocimientos, actitudes y prácticas sobre el manejo de enfermedades diarreicas agudas en las madres de niños menores de 4 años del Centro Poblado Virgen del Carmen - La Era, 2013

de la intervención una media de 27,53 lo cual indica que las madres o cuidadoras mejoraron la forma en que manejaban los episodios de EDAS. Los estudios realizados por Mendigure (2007) son similares a los nuestros ya que el programa mejoró las prácticas en el manejo de EDAS. Los resultados obtenidos antes de la intervención del programa, en las prácticas de las madres, fueron que el $80 \%$ no presentaba las manos limpias y uñas cortadas. No obstante, luego de la implementación del programa se aprecia que solo el $10 \%$ de las madres no practica estas medidas de higiene. De manera similar ocurre con la mayoría de las prácticas que previenen la aparición de las EDAS.

\section{Declaración de financiamiento y de conflicto de} intereses:

El estudio fue financiado por la autora, quien declara no tener algún tipo de conflicto de interés en la investigación realizada.

\section{Correspondencia:}

Marisol Sotelo Rodríguez

Universidad Peruana Unión

Carretera Central Km. 19.5 Naña

Correo electrónico:

marsol_2906@hotmail.com

\section{REFERENCIAS BIBLIOGRÁFICAS}

Benguigui.Y, Bossio J. Fernández, H. (2001). Investigaciones operativas sobre atención integrada a las enfermedades prevalentes de la infancia OPS. Washington DC: Recuperado de http://www.searchdocument.com/pdf/3/12/tasa-de-mortalidad-infantil. html

Cabrera, Tascón, Lucumí (2001). Creencias en salud: historia, constructos y aportes del modelo. Revista Facultad Nacional Pública. http://www.bvsde.paho. org/bvsacd/cd26/fulltexts/0766.pdf

Cabezas (2011). "Intervención educativa sobre prevención de enfermedades diarreicas agudas desde el enfoque AIEPI comunitario para madres con niños menores de cinco años del centro de educación inicial Lic. Alfonzo Chávez Jara - Ecuador" recuperado de dspace.spach. edu.ec/handle/123456789/2042.

Instituto Nacional de Estadística (2003). Niveles, tendencias y diferenciales de la mortalidad infantil. Perú Recuperado de http://www.ine.gov.bo/PDF/ MortalidadInfantil/Infantil0.pdf. http://www.searchdocument.com/pdf/3/12/tasa-de-mortalidad-infantil. html
Mendigure, J. Huamán, S. Ramos, N. Valencia, E. (2007). "Efectividad de un programa de intervención educativa para La disminución de diarreas infantiles en Lima, Perú" recuperado de posgrado.upeu.edu.pe/revista/ filev2/87-100.pdff.

MINSA - Dirección General de Salud de las Personas (2011). Proyecto Vigía. Estudio de etiología de la diarrea en las direcciones de salud Cajamarca, Lambayeque, Loreto y Lima Este. Recuperado de http:// www.ins.gob.pe/repositorioaps/0/4/jer/cnspresanti documentos técnicos/ E20studio etiológico diarrea_4 DISAS.pdf

Quispe (2012). Conocimientos, actitudes y prácticas de las madres con niños menores de 5 años con casos de EDAS de la comunidad de Yuncaypata que acuden al centro de salud de Wanchaq Cusco, recuperado de http://www.slideshare.net/josesequeiros5/proyecto-deinvestigacion-final-eda.

Recibido: 19/04/2014 Aceptado: 26/09/2014 